\title{
Magnitude and Phase Response of High Pass FIR filter using Rectangular, Hamming and Bartlett Window Techniques
}

\author{
Durgesh Sahu ${ }^{1}$, Mukesh Chandra ${ }^{2}$, Pranay Kumar Rahi ${ }^{3}$ \\ B.E Scholar, Dept of Electrical and Electronics Engineering, Institute of Technology, Korba, Chhattisgarh, India ${ }^{1,2}$ \\ Assistant Professor, Dept of Electrical and Electronics Engg, Institute of Technology, Korba, Chhattisgarh, India ${ }^{3}$
}

\begin{abstract}
A Digital Signal Processing is a main branch of electronics. In the digital control system, which is mixed in the input signal, has a great influence on the performance of the system. Therefore, processing of input signal has to be done to get useful signal. It is concerned with the representation by sequence of number or symbol and the processing of these signals. DSP have many more applications which are useful in our life i.e military processing of these signals. DSP have many more radar signal processing, sonar signal processing, navigation, instrument and control spectrum analysis etc. Signal filtering is the main application of DSP. In signal processing, a filter is a device or process that removes some unwanted components or features from a signal. In this we discuss about High Pass FIR filter using Rectangular, Hamming and Bartlett window techniques. By comparative analysis of these window techniques we conclude that Rectangular window technique is better in comparison with them techniques. In this technique the maximum wanted signals are present after cut off frequency.
\end{abstract}

Keywords: DSP, Digital Filter, FIR filter, Blackman, Rectangular, MATLAB.

\section{INTRODUCTION}

Digital signal processing is the technique employed for the mathematical manipulation of an information signal so as to modify or improve it. For this purpose filters are mainly used. Digital filters are used in numerous applications e.g. control system, system for audio and video processing and communication system. Digital filters can be applied to very low frequency signals, such as those occurring in biomedical and seismic applications very efficiently.[1] Most signals we encounter are generated by natural means. However, a signal can also be generated synthetically or computer simulation. A signal carries information, and the objective of signal processing is to extract useful information carried by the signal. The method of information extractions depends on the type of signal and the nature of the information being carried by the signal. Thus, roughly speaking signal processing is concerned with the mathematical representation of the signal and the algorithmic carried out on it to extract the information present. The representation of the signal can be in terms of basic functions in the domain of the original independent variable(s), or it can be in terms of basis function in a transform domain. Likewise, the information extraction process may be carried out in the original domain of the signal or in transform domain [2].

There are two major types of digital filters are:

1) Infinite Impulse response (IIR) filters

2) Finite Impulse response (FIR) filters

Infinite Impulse Response (IIR) digital filter has the problems of phase non-linearity. Therefore it is a low order filter which becomes highly unstable. Due to these factors, the FIR filter can be used to design a linear phase digital filter which is convenient for image processing and data transmission applications. The FIR filters are broadly used in various fields, such as long distance communication, image processing applications etc The system function of FIR filter is given below:

$$
H(z)=\sum_{n=0}^{L-1} h(n) z^{-n}
$$

where, $\mathrm{L}$ is the length of the filter, and $\mathrm{h}[\mathrm{n}]$ is the impulse response.[3]

\section{WINDOW TECHNIQUE}

The FIR filter design process using window function can be enumerated as

1.Define filter specification.

2. Specify a window function according to the filter specification.

3. Compute the filter order required for a given set of specification.

4. Compute the coefficients of the window function to be used.

5. Compute the coefficients the ideal filter according to the filter order.

6. Compute FIR filter coefficients in accordance the obtained window function and the coefficients of the ideal filter. 
7. If the resulting filter has a very wide or a very narrow transition region, it is mandatory to change the filter order by decreasing it according to needs, and after this process the steps 4,5 and 6 are interated as many times as needs.[4]

In this paper the window techniques are used to design the FIR filter, which are

1.Rectangular

2.Hamming

3.Bartlett

\section{WINDOWS AND THEIR EQUATIONS}

Most digital signals are infinite, or sufficiently large that the data set cannot be manipulated as a whole. Sufficiently large signals are also difficult to analyze statically, because statistical calculation require all points to be available for analysis. In order to avoid these problems, engineers typically analyze small subsets of the total data, through a process called windowing. The window design method does not produce filters that are optimal (in the sense of meeting the design specifications in the most computationally efficient fashion), but the method is easy to understand and does produces filters that are reasonably good. Off all the hand design methods the window method is the most popular and effective[2]

Window can be categorized as fixed or adjustable window function. Fixed window such as rectangular, hanning, hamming, and blackman window have only one independent parameter window length which control the main-lobe width. Adjustable window have two or more independent parameters such as window length and one more additional parameters that can control the other window's characteristics [6,7]. The Kaiser window is a kind of two parameter window function. In a Kaiser window width of main lobe can be controlled by adjusting the length of the filter and side lobe level can be controlled by varying the other parameter. But the Kaiser window has the disadvantage of higher computational complexity due to the use of vessel function in the calculation of the window coefficient.

However the amplitude of side lobe and ripples in pass band is also increased. The function is

$$
\omega(n)=\left\{\alpha-(1-\alpha) \cos \left(\frac{2 \pi n}{M-1}\right), 0 \leq n \leq M-1\right.
$$

Otherwise the value of $(n)$ is zero.

\subsection{RECTANGULAR WINDOW}

The rectangular window (sometimes known as the 32 Tboxcar T0TorT32T Dirichlet (0Twindow) is the simplest window, equivalent to replacing all bu0T 0TNOT 0Tvalues of a data sequence by zeros, making it appear as though the waveform suddenly turns on and off: Other windows are designed to moderate these sudden changes, which reduces scalloping loss and improves dynamic range. The rectangular window is the 1st order0T 0TBspine window as well as the 0th power0T 0T32Tcosine window.[8]

$$
W_{R}(n)=\left\{\begin{array}{c}
1 \text { for }|n| \leq \frac{M-1}{2} \\
0, \text { otherwise }
\end{array}\right.
$$

\subsection{Hamming Window}

Hamming window has a bell-like shape. Its first and last samples are not zero. The window is optimized to minimize the maximum side lobe.[5]

$$
\begin{aligned}
& \mathrm{W}(\mathrm{n})=0.54-0.46 \cos \frac{2 \pi \mathrm{n}}{\mathrm{N}-1}, 0 \ll \mathrm{N} \ll \mathrm{L}-1 \\
& \quad=0 \quad \text {, otherwise }
\end{aligned}
$$

\subsection{Bartlett Window}

The window function of a non-causal Bartlett window is expressed by

$$
\mathrm{W}_{\text {Bar }}(\mathrm{n})=\left\{\begin{array}{c}
1+\mathrm{n},-\frac{\mathrm{M}-1}{2}<\mathrm{n}<1 \\
1-\mathrm{n}, 1<\mathrm{n}<\frac{\mathrm{M}-1}{2}
\end{array}\right.
$$

\section{DESIGN SIMULATION}

Table 2.1 Filter parameters and value

\begin{tabular}{|l|c|}
\hline \multicolumn{1}{|c|}{ PARAMETER } & VALUE(Hz) \\
\hline Sampling frequency $\left(\mathrm{f}_{\mathrm{s}}\right)$ & 45000 \\
\hline Cut off frequency $\left(\mathrm{f}_{\mathrm{c}}\right)$ & 10800 \\
\hline
\end{tabular}

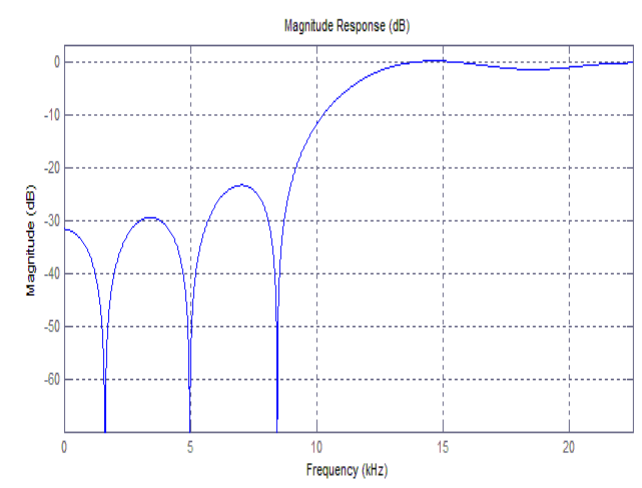

Fig2.1 Magnitude response of Rectangular window Technique

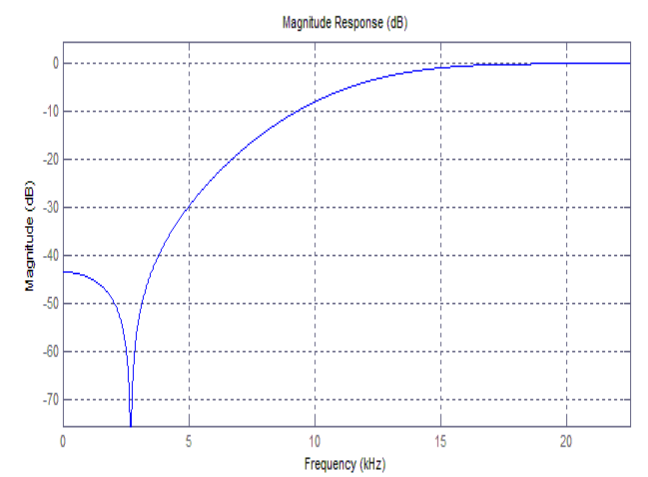

Fig2.2 Magnitude Response of Hamming window Technique 


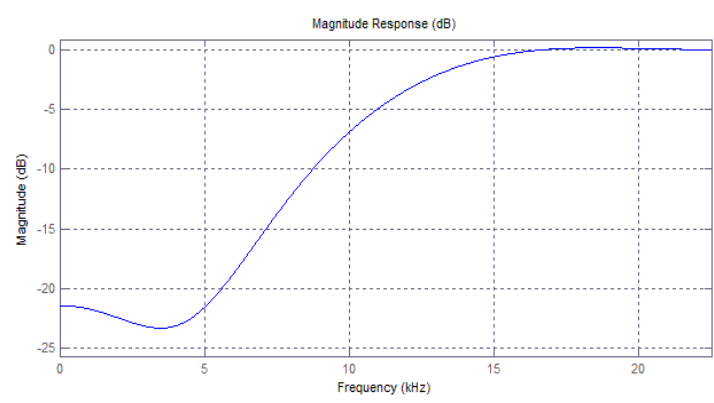

Fig 2.3 Magnitude Response of Bartlett Window Technique

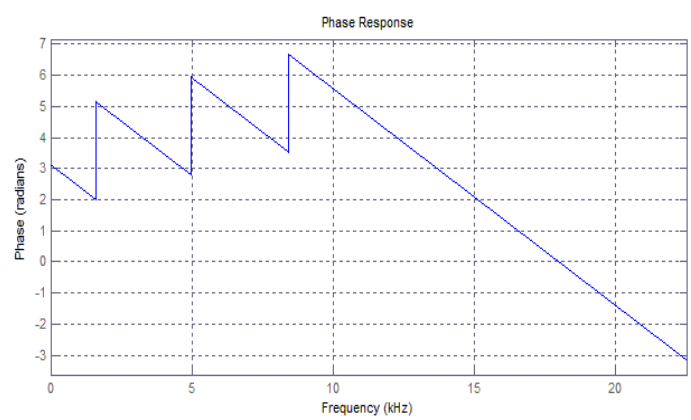

Fig 2.4 Phase Response of Rectangular Window Technique

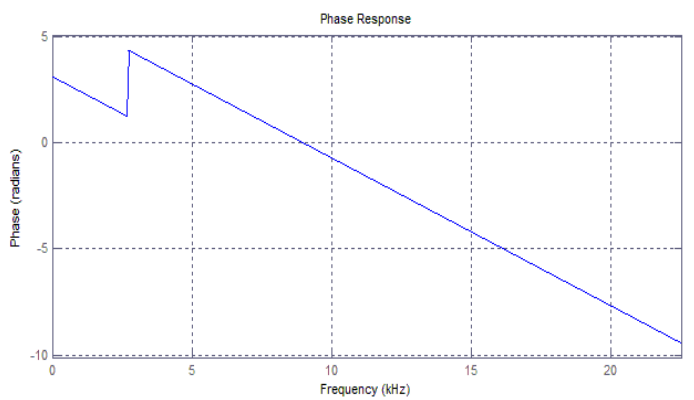

Fig 2.5 Phase Response of Hamming Window Technique

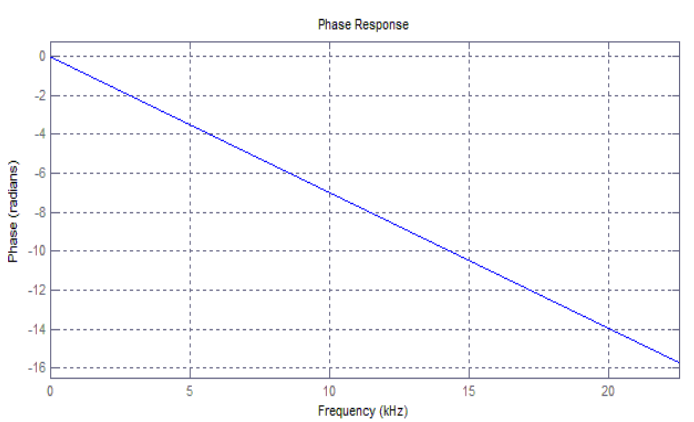

Fig 2.6 Phase Response of Bartlett Window Technique

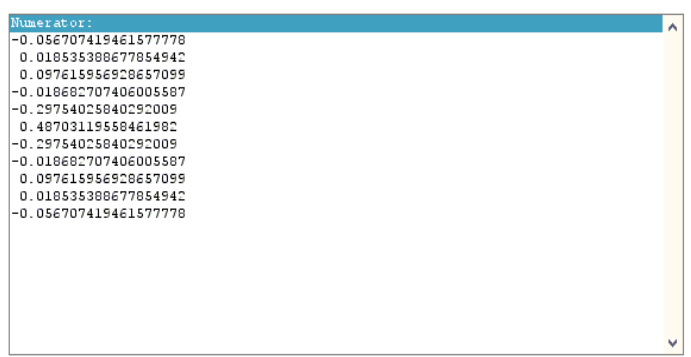

Fig 2.7 Filter Cofficient for Rectangular Window Technique

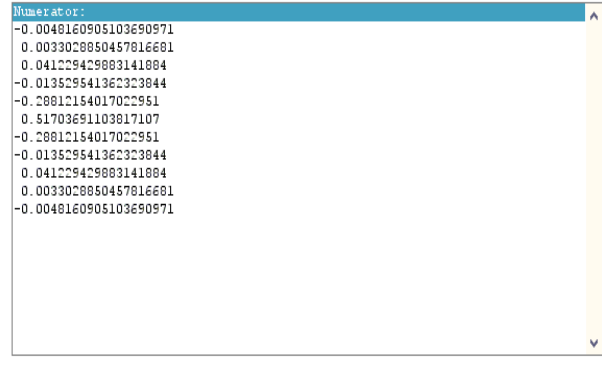

Fig 2.8 Filter Cofficient of Hamming Window Technique

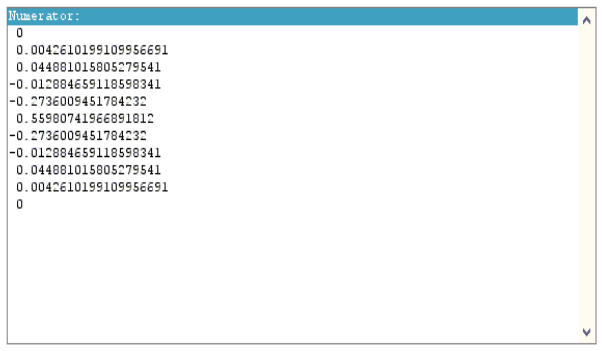

Fig 2.9 Filter Cofficient of Bartlett Window Technique
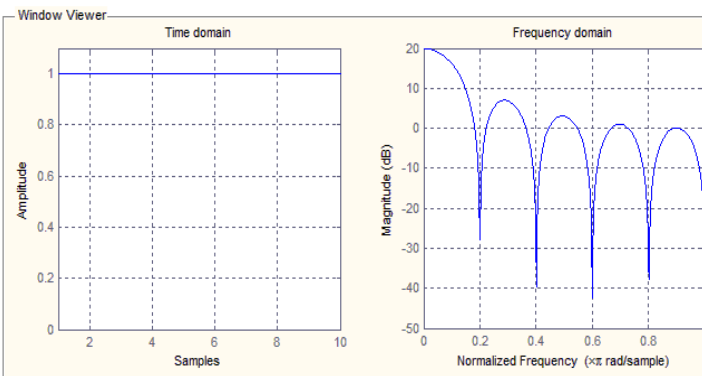

Fig 2.10 Time Domain and Frequency Domain of Rectangular Window

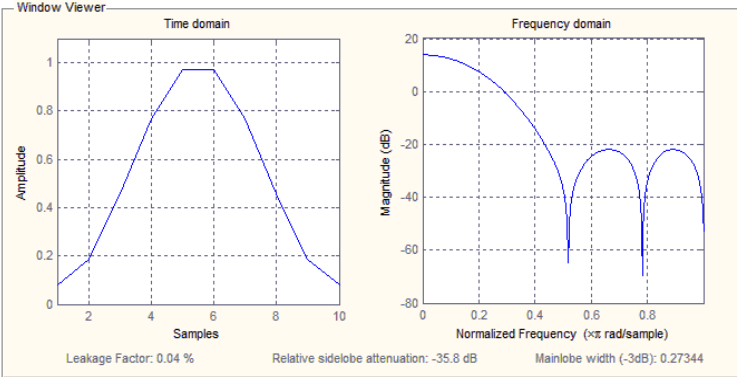

Fig 2.11 Time Domain and Frequency Domain of Hamming Window
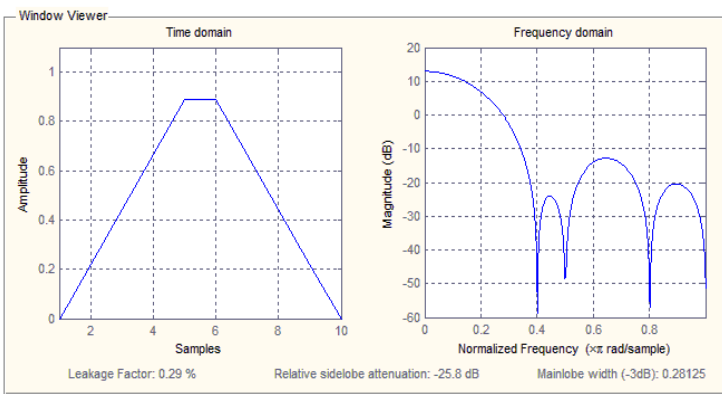

Fig 2.12 Time Domain and Frequency Domain of Bartlett Window 


\section{COMPARITIVE ANALYSIS}

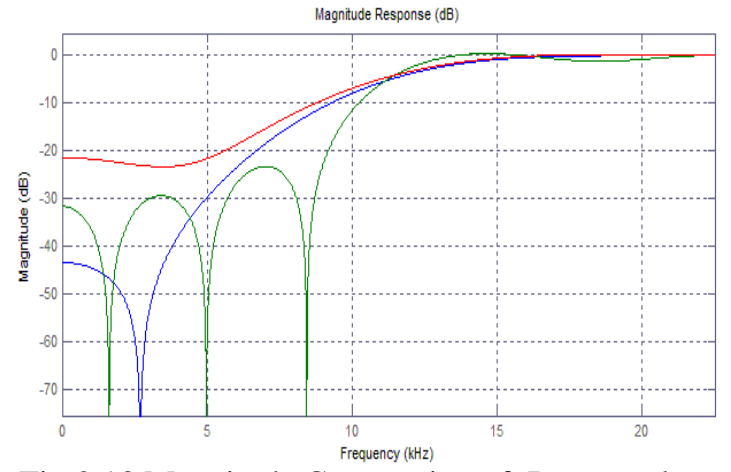

Fig 2.13 Magnitude Comparsion of Rectangular, Hamming and Bartlett Window

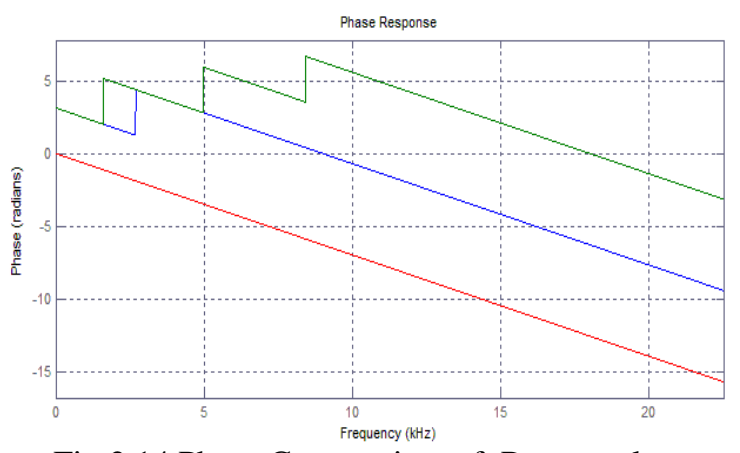

Fig 2.14 Phase Comparsion of Rectangular, Hamming and Bartlett Window

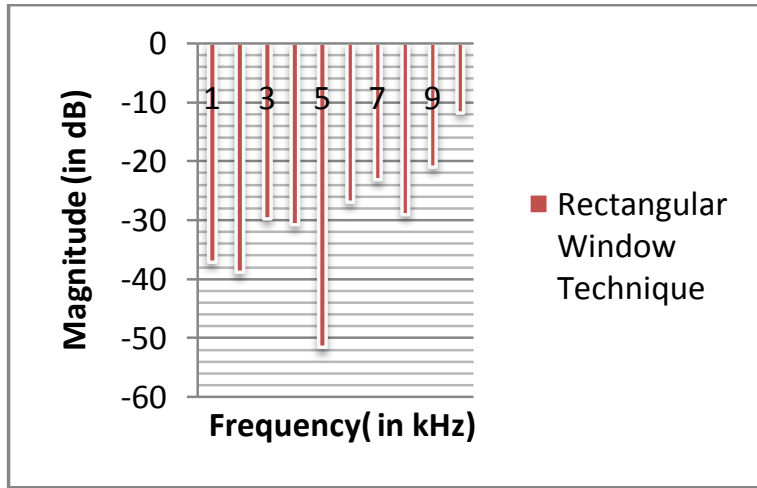

Chart 1.1 Magnitude and Frequency plot of Rectangular window

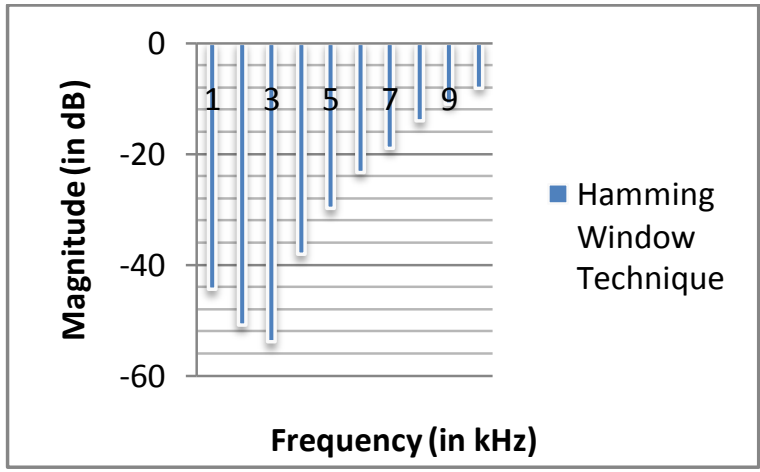

Chart 1.2 Magnitude and Frequency plot of Hamming window

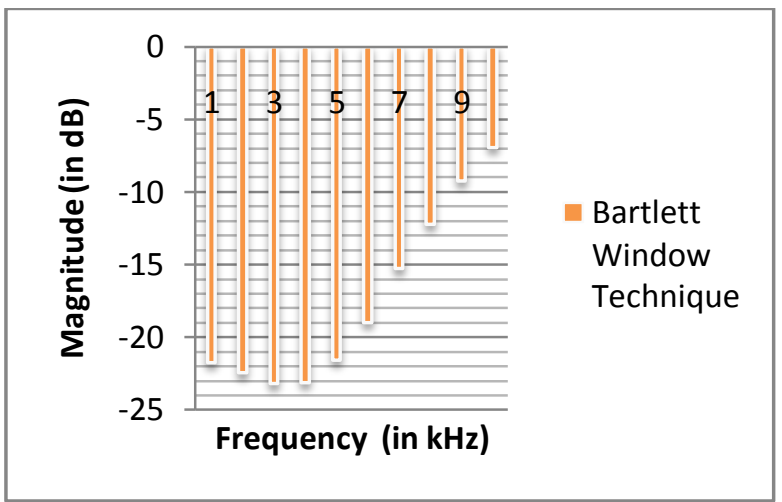

Chart 1.3 Magnitude and Frequency plot of Bartlett window

\section{RESULT}

Table 2.2 Simulation result from Matlab

\begin{tabular}{|l|l|l|l|}
\hline $\begin{array}{l}\text { Window } \\
\text { Technique }\end{array}$ & $\begin{array}{l}\text { Relative } \\
\text { sidelobe } \\
\text { attenuation }\end{array}$ & $\begin{array}{l}\text { Mainlobe } \\
\text { width } \\
(-3 \mathrm{~dB})\end{array}$ & $\begin{array}{l}\text { Leakage } \\
\text { factor }\end{array}$ \\
\hline $\begin{array}{l}\text { Rectangul } \\
\text { ar window }\end{array}$ & $-13 \mathrm{~dB}$ & 0.17188 & $9.2 \%$ \\
\hline $\begin{array}{l}\text { Hamming } \\
\text { window }\end{array}$ & $-25.8 \mathrm{~dB}$ & 0.28125 & $0.29 \%$ \\
\hline $\begin{array}{l}\text { Bartlett } \\
\text { window }\end{array}$ & $-35.8 \mathrm{~dB}$ & 0.27344 & $0.04 \%$ \\
\hline
\end{tabular}

Table 2.3 Magnitude and Frequency results of Rectangular, Hamming and Bartlett Window

\begin{tabular}{|l|l|l|l|}
\hline Frequency $(\mathrm{kHz})$ & \multicolumn{4}{|l|}{ Magnitude $(\mathrm{dB})$} \\
\hline 1 & -37.1348 & -44.3371 & -21.7389 \\
\hline 2 & -38.8753 & -50.7706 & -22.4330 \\
\hline 3 & -29.7796 & -53.7869 & -23.1806 \\
\hline 4 & -30.7898 & -37.9877 & -23.0954 \\
\hline 5 & -51.6209 & -29.7829 & -21.5494 \\
\hline 6 & -26.9530 & -23.2196 & -18.9634 \\
\hline 7 & -23.2130 & -18.8474 & -15.2730 \\
\hline 8 & -29.0528 & -13.9895 & -12.2233 \\
\hline 9 & -21.0490 & -10.8267 & -09.2495 \\
\hline 10 & -11.8508 & -8.0326 & -06.9405 \\
\hline
\end{tabular}

From MATLAB simulation result of Rectangular, Hamming and Bartlett window technique at sampling frequency $\left(f_{s}\right) 45000 \mathrm{~Hz}$ and cut-off frequency $\left(f_{c}\right) 10800$ Hz.

\section{CONCLUSION}

In this research paper High pass FIR filter has been designed using MATLAB Rectangular, Hamming and Bartlett window technique. It concludes by comparative values of both magnitude and phase response of the filter using both the techniques at same frequency i.e. 
In Rectangular windowing technique wanted signals are present in maximum after the cut-off frequency.

In Hamming windowing technique the unwanted signals are not present after the cut-off frequency, but this technique also cuts the wanted signals.

In Bartlett windowing technique wanted signals are present in maximum after the cut-off frequency but some unwanted signals are also present with the wanted signals too.

\section{REFERENCES}

[1]. Rahul Kurrey, Versha Nirala and Pranay Kumar Rahi "Optimal Design of Low Pass Filter By Parzen, Taylor and Hamming Technique" IJRASET, Vol. No.5, Issue No.II, February 2017 ISSN: 2321-9653.

[2]. Pooja Pandey, Sumit Chakravorty, Durgesh Sahu and Pranay Kumar Rahi "Magnitude and Phase Response of Low Pass FIR Filter using Rectangular and Blackman Window Technique" IJISET, Vol. No.3, Issue No.8, Auguest 2016 ISSN(Online)23487968.

[3] Mohd. Shariq Mahoob and Rajesh Mehera "Design of low pass FIR filter using Hamming Blackman-Harris and Taylor window" IJARSE, Vol. No.3, Issue No.11, November 2014 ISSN23198354(E).

[4]. Pramod Yadav, Shankarlal Sahu, Vijendra Prajapati "DESIGNING A LOW PASS FIR FILTER BY USING RECTANGULAR AND TALOR WINDOW" IJISET, Vol. No.3, Issue No.8,Auguest 2016,ISSN(Online)2348-7968.

[5]. J.R.Johnson, "Introductiont to Digital Signal Processing,"4th ed., Prentice-Hall of India Private Limited, 1997, pp. 85-95

[6]. T. Saramaki, Finite impulse response filter design in Handbook for Digital Signal Processing, Edited by S. K. Mitra and J. F. Kaiser, IBSN 0-471-61995-7 John Wiley andSons Inc, 1993.

[7]. Oppenheim, R. Schafer, and J. Buck, DiscreteTime Signal Processing second edition, PrenticeHall,1999

[8]. https://en.wikipedia.org/wiki/Window_function.

\section{BIOGRAPHIES}

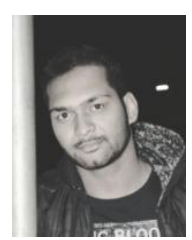

Durgesh Sahu pursuing Bachelor of Engineering in Electrical and Electronics Engineering, in $6^{\text {th }}$ semester from Institute of Technology, Korba, affiliated from Swami Vivekanand Technical University, Chhattisgarh, India.

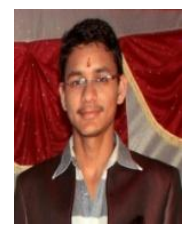

Mukesh Chandra pursuing Bachelor of Engineering in Electrical and Electronics Engineering, in $6^{\text {th }}$ semester from Institute of Technology, Korba, affiliated from Swami Vivekanand Technical University, Chhattisgarh, India.

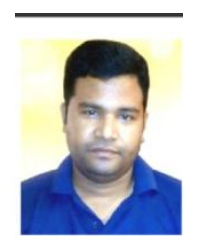

Pranay Kumar Rahi received the Bachelors of Engineering degree in Electronics and Telecommunication Engineering from Government Engineering College, Guru Ghasidas University, Bilaspur, Chhattisgarh, India in 2004, and pursuing Masters of Engineering in Electronics and Communication Engineering from National Institute of Technical Teacher's Training and Research, Punjab
University, Chandigarh, India. Working as a Assistant professor in Electrical and Electronic Engineering Department of Institute of Technology, Korba since 2008. He has authored 14 research publications and published a number of Journal papers and research paper in the leading International and National Journal. His primary research interest includes Digital Signal Processing, VLSI Design, Control System and Digital Electronics and logic design. 